\title{
A FIXED POINT THEOREM FOR MAPPINGS IN SCALED METRIC SPACES, WITH APPLICATIONS TO PARTIAL DIFFERENTIAL EQUATIONS
}

\author{
BY P. C. ROSENBLOOM ${ }^{1}$
}

Communicated by François Treves, October 27, 1972

\begin{abstract}
A fixed point theorem is given for mappings of scaled metric spaces. This theorem applies to the Cauchy problem for partial differential equations, to singular differential equations, and to the Goursat problem.
\end{abstract}

1. Introduction. In this note we give a fixed point theorem for a class of mappings of a space with a two-parameter scale of metrics. This theorem contains as special cases the classical Cauchy-Kovalevska theorem (see Rosenbloom [6], [7]), as well as the generalizations of Ovsjannikov [3], Treves [9], and Duchateau and Treves [1]. It can also be applied to some classes of partial differential equations such as those treated by Rosenbloom [8], to the Goursat problem, and to other cases of the Riquier problem (see [4], [5]).

We shall say that $\left\{d_{r, s} \mid r, s \in(0,1]\right\}$ is a scale of metrics on the space $S$ if each $d_{r, s}$ is a metric on $S$, and $d_{r, s}$ is nondecreasing in each of the variables $r$ and $s$. If $A$ is a subset of $S$, then we denote by $A_{r, s}$ the completion of $A$ with respect to $d_{r, s}$. For $\alpha>0$, we shall denote by $\delta_{r, s}^{\alpha}$ the metric

$$
\delta_{r, s}^{\alpha}(u, v)=r^{\alpha} \sup _{0<\rho \leqq r} \rho^{-\alpha} d_{\rho, s}(u, v) .
$$

Let $c$ be a function on $R^{+}=\{x \mid x>0\}$ to $R^{+}$, let $\mu, v \in R^{+}$. We shall say that $T$ is a mapping of class $K(c, \mu, v)$ if, for $0<r \leqq 1,0<s<\sigma \leqq 1, T$ is a mapping of $S_{r, \sigma}$ into $S_{r, s}$ and satisfies

$$
d_{r, s}(T u, T v) \leqq \frac{c(n) r^{\mu}}{c(n+\mu)(\sigma-s)^{v}} \delta_{r, \sigma}^{n}(u, v)
$$

for $u, v \in S, n>0$. For any $\lambda>0$ we denote by $\Delta_{\lambda}$ the metric

$$
\Delta_{\lambda}=\sup \left\{d_{r, s} \mid r^{\mu} \leqq \lambda(1-s)^{\nu}\right\}
$$

and by $A(\lambda)$ the completion of the set $A$ with respect to $\Delta_{\lambda}$.

THEOREM. Suppose that $\left\{d_{r, s}\right\}$ is a scale of metrics on $S$ and $T$ is a mapping of class $K(c, \mu, v)$, and that the power series

AMS (MOS) subject classifications (1969). Primary 3503, 3536, 3537.

Key words and phrases. Cauchy-Kovalevska, Cauchy problem, scale of metrics, singular differential equations, semigroups, Goursat problem.

${ }^{1}$ This research was supported by NSF research grant 29704. 


$$
F(\lambda)=c(\alpha) \sum_{n=0}^{\infty} n^{n v} \lambda^{n} / c(\alpha+n \mu)
$$

has the radius of convergence $R, 0<R \leqq+\infty$. Then, for any $\alpha>0$ and any $u_{0} \in S$ such that $\beta_{0}=\delta_{1,1}^{\alpha}\left(T u_{0}, u_{0}\right)<+\infty$, there is a fixed point $u$ of $T$ in $\bigcap\{S(\lambda) \mid \lambda<R\}$ such that $\delta_{r, s}^{\alpha}\left(u, u_{0}\right) \leqq \beta_{0} \lambda^{\alpha / \mu} F(\lambda)$ for $r^{\mu} /(1-s)^{v}=\lambda<R$. There is no other fixed point $v$ in any of the spaces $S(\lambda), \lambda<R$, such that $\delta_{r, s}^{a}\left(v, u_{0}\right)<+\infty$ for some $r, s \in(0,1]$.

2. Sketch of proof. Let $u_{n}=T^{n} u_{0}$. If $r^{\mu} /(1-s)^{v}=\lambda$, then we can easily prove by induction that

$$
d_{r, s}\left(u_{n+1}, u_{n}\right) \leqq \beta_{0} c(\alpha) n^{n v} r^{\alpha} \lambda^{n} / c(\alpha+n \mu)
$$

for $n \geqq 0$. We use (1) with $\sigma=(1+n s) /(1+n)$. The convergence of $u_{n}$ to a fixed point $u$, in the metric $\Delta_{\lambda}, \lambda<R$, and the above estimates now follow.

Let $T^{\infty}$ be the mapping defined by $T^{\infty} u=\lim _{n \rightarrow \infty} T^{n} u$. Then $T^{\infty}$ is defined on the set $A_{\alpha}=\left\{u \mid u \in S, \delta_{1,1}^{\alpha}(T u, u)<+\infty\right\}$, and maps $A_{\alpha}$ into $\bigcap\{S(\lambda) \mid \lambda<R\}$. The same inductive argument shows that if $v \in A_{\alpha}$, $\delta_{1,1}^{\alpha}(u, v)<+\infty$, then $\Delta_{\lambda}\left(T^{\infty} u, T^{\infty} v\right)=0$. Hence the equivalence relation

$$
u \equiv{ }_{\alpha} v \leftrightarrow \delta_{1,1}^{\alpha}(u, v)<+\infty
$$

divides $A_{\alpha}$ into equivalence classes each of which is associated with a unique fixed point $T^{\infty} u$ of $T$. The last assertion can be obtained by considering the scale $\tilde{d}_{r, s}=d_{\text {ar }, b s}$ for any $a, b \in(0,1]$.

The equivalence class of $u$ in $A_{\alpha}$ is a "boundary value" of $u$, of order $\alpha$ with respect to the given scale on $S$.

3. The Cauchy-Kovalevska theorem. Let $\Omega$ be a domain in $C^{N}$, let $\delta(x)$ be the distance from $x$ to the complement of $\Omega$, and suppose that $\delta(x)$ $\leqq k<+\infty$ for all $x \in \Omega$. Let $S$ be the space of continuous functions on the interval $I=[0,1]$ to $B(\Omega)$, the space of bounded analytic functions on $\Omega$ to $C^{M}$. We set $d_{r, s}(u, v)=\|u-v\|_{r, s}$, where

$$
\|u\|_{r, s}=\sup \{|u(t, x)| \mid 0 \leqq t \leqq r, \delta(x)>(1-s) k\} .
$$

Then $d_{r, s}$ is a scale of metrics on $S$. We may take

$$
(T u)(t, x)=\Gamma(\mu)^{-1} \int_{0}^{t}(t-\tau)^{\mu-1}(L u)(\tau, x) d \tau,
$$

where $L$ is a linear differential operator of order $v$ from $B(\Omega)$ to $B(\Omega)$ with coefficients continuous on $I$. Then $T$ is of class $K(c, \mu, v)$ for a certain $c$, and (2) is satisfied if $v \leqq \mu$. If $\mu$ is a positive integer, then we obtain the classical Cauchy-Kovalevska theorem.

As in [7] we may replace $C^{N}$ and $C^{M}$ by general Banach spaces $X$ and $U$. 
We remark that if $L$ is an analytic partial differential and $S$ is any Banach space of analytic functions on $\Omega$ to $U$ and the scale is defined in terms of norms on $S$, then our condition (1) on $T$ (or the Duchateau-Treves condition (1.5)) implies that $L$ is linear. Hence neither our present theorem nor that of Duchateau and Treves [1] contains directly the theorems for nonlinear analytic equations given in [7], [8], and [9]. If we only assume that $T$ satisfies (1) in some ball such as $\left\{u \mid \Delta_{\lambda}\left(u, u_{0}\right) \leqq 1\right\}$, then the inductive proof of (3) fails since the choice of $\sigma$ may take us out of the set on which (1) holds. Our version of the majorant method in [7] (which can be made more abstract as done by Treves [9]) yields a space $S$ which is transformed into itself by $T$, and this implies that $T$ satisfies (1) on $S$ (see the proof of Theorem 2 in [7]). It is not obvious how such a result can be obtained without some variant of the majorant method. Of course, if we use scales defined in terms of such spaces of $C^{\infty}$ functions as the Gevrey classes, then we can give genuinely nonlinear operators which satisfy (1), as is done in [1].

4. Singular partial differential equations. In [8] we showed that a quite general linear equation with a regular singularity at $t=0$ can be reduced to the form

$$
t u_{t}-B(x) u=t L u, \quad u(0, x)=0,
$$

where

$$
L u=D(t, x)+E(t, x) u+C(t, x) u_{x} .
$$

We shall assume that $B$ is analytic and bounded in a domain $\Omega \subset X$, where $X$ is a Banach space, with values in $P=U^{U}$. (We shall denote by $U^{V}$ the space of bounded linear transformations of $V$ to $U$.) We shall assume that $D, E$, and $C$ are analytic and bounded on $\Omega_{K}=C_{K} \times \Omega, C_{K}=\{t \mid$ $|t|<K\}, K>1$, with values in $U, P, U^{V}$, where $V=U^{X}$. If $g(t, x)$ $=\sum_{n=1}^{\infty} a_{n}(x) t^{n-1}$ is analytic on $\Omega_{K}$ to $U$, then the solution operator $u=\wedge g$ of

$$
t u_{t}-B(x) u=t g, \quad u(0, x)=0
$$

is

$$
u(t, x)=\sum_{n=1}^{\infty}(n I-B(x))^{-1} a_{n}(x) t^{n}
$$

We shall assume that, for some $b$ and all $x \in \Omega, n>0$,

$$
\left\|(n I-B(x))^{-1}\right\| \leqq \beta / n \text {. }
$$

(This is true if the distance from the spectrum of $B(x)$ to the set of positive integers is bounded away from zero.) We may define $T=\wedge L$. We can take $S$ as the space of bounded analytic functions $u$ on $\Omega_{1}$ to $U$, and define 


$$
\begin{aligned}
& d_{r, s}(u, v)=\|u-v\|_{r, s}, \text { where, for } u=\sum a_{n}(x) t^{n}, \\
& \|u\|_{r, s}=\sup \left\{r^{n}\left\|n_{n}(x)\right\| \mid n \geqq 0, \delta(x)>(1-s) k\right\} .
\end{aligned}
$$

It is easy to verify that $T \in K(c, 1,1)$ with $c(n)=b^{n} \Gamma(n+1)$ for a certain $b>0$, so that $R=b / e$.

These conditions also apply to many cases where $B(x)$ is an unbounded operator. Suppose $B(x)$ generates a strongly measurable semigroup $\{\Phi(\tau, x)\}$ of type $\omega(x)$, uniformly bounded on $\Omega$ (see Hille and Phillips [2]) such that $\Phi(\tau, x) U \subset D(B(x))$ for $\tau>0, x \in \Omega$. Suppose $L$ is as above with coefficients continuous in $t$ for $t \geqq 0$ and analytic in $\Omega$, and suppose they satisfy inequalities of the form

$$
\|D(t, x)\| \leqq e^{-\alpha t} M \quad \text { for } t \geqq 0, x \in \Omega,
$$

for some $\alpha>\sup _{x \in \Omega} \omega(x)$. Then the transformation

$$
(T u)(t, x)=\int_{t}^{\infty} \Phi(\tau-t, x)(L u)(\tau, x) d \tau
$$

satisfies (1) for a suitable scale of metrics. The fixed point $u=T^{\infty} 0$ is the unique solution of

$$
\partial u / \partial t=B(x) u-L u
$$

satisfying $\|u(t, x)\|=O\left(e^{-\alpha t}\right)$ as $t \rightarrow+\infty$, and is analytic in a domain of the form $\lambda<e^{t} \delta(x)$ for a certain positive constant $\lambda$.

We leave the discussion of the Goursat problem to a fuller report of our work, now in preparation.

\section{BIBLIOGRAPHY}

1. P. Duchateau and F. Treves, An abstract Cauchy-Kovalevska theorem in scales of Gevrey classes, Inst. Naz. Alta Mat. Sympos. Math. 7 (1971), 135-163.

2. E. Hille and R. S. Phillips, Functional analysis and semi-groups, rev. ed., Amer. Math. Soc. Colloq. Publ., vol. 31, Amer. Math. Soc., Providence, R.I., 1957. MR 19, 664.

3. L. V. Ovsjannikov, Singular operator in a scale of Banach spaces, Dokl. Akad. Nauk SSSR 163 (1965), 819-822 = Soviet Math. Dokl. 6 (1965), 1025-1028. MR 32 \#8164.

4. C. Riquier, La méthode des fonctions majorantes et les systèmes d'équations aux dérivées partielles, Mém. Sci. Math., fasc. 32, Gauthier-Villars, Paris, 1928.

5. J. F. Ritt, Differential algebra, Amer. Math. Soc. Colloq. Publ., vol. 33, Amer. Math. Soc., Providence, R.I., 1950. MR 12, 7.

6. P. C. Rosenbloom, Linear partial differential equations, Surveys in Appl. Math., vol. V, Wiley, New York, 1958, pp. 45-196.

7. P. C. Rosenbloom, The majorant method, Proc. Sympos. Pure Math., vol. 4, Amer. Math. Soc., Providence, R.I., 1961, pp. 51-72. MR 26 \#2713.

8. - Singular partial differential equations, Proc. Sympos. Fluid Dynamics and Appl. Math. (Univ. Maryland, 1961), Gordon and Breach, New York, 1962, pp. 67-77. MR 28 \# 3234.

9. F. Treves, An abstract nonlinear Cauchy-Kovalevska theorem, Trans. Amer. Math. Soc. 150 (1970), 77-92.

Department of Mathematics, Columbia University Teachers College, New York, NEW YORK 10027 